\title{
Modeling of Atmospheric Transmission Coefficient in Infrared for Thermovision Measurements
}

\author{
Minkina Waldemar, ${ }^{1,{ }^{*}}$ Klecha Daniel $^{1}$ \\ ${ }^{1}$ Czestochowa University of Technology, Al. Armii Krajowej 17, \\ PL 42-200 Czestochowa, Poland \\ *Corresponding author: minkina@el.pcz.czest.pl
}

\begin{abstract}
The aim of this paper is to discuss different models that describe atmospheric transmission in infrared. They were compared in order to choose the most appropriate one for certain atmospheric conditions. Universal models and different inaccuracies connected with them were analyzed in this paper.
\end{abstract}

Key words: Infrared thermography, atmospheric transmission, atmospheric conditions, numerical simulation, thermal imaging.

\section{Introduction}

The atmosphere transmission in infrared is an important parameter in thermovision measurements. This is due to the fact that when the temperature of an object is measured the atmosphere which is between the thermal imaging camera and the object blocks infrared radiation emitted by the object. Correctly measured atmospheric transmission allows to determine the correct temperature of the object. In the case when there was no precise model describing the microcontroller in the thermal imager [1], the temperature of the object would be wrong, lower or higher.

The paper compares different methods of calculating of the atmospheric transmission coefficient in infrared which can be found in practice and in literature. When a model is chosen such factors as accuracy and the time needed to do the measurements should be taken into consideration. In fact, that subject of research about the atmosphere transmission have wide range, this paper has some limitations. The paper concentrate only on the atmosphere impact on the measurement. The effect of the IR radiation emitted by the absorbing atmosphere (Kirchhoff's law) was skipped in this case and will be considered in next paper. Thermal imaging cameras operate in a particular infrared range for which the atmospheric transmission coefficient will be different than for the whole band. Graphs transmission factor atmosphere for cameras having different specifications and matrices (for example, microbolometer arrays with flat spectral response or MCT arrays) will never be the same. Bearing in mind described limitations, the paper shows only atmospheric transmission waveforms for different models and the differences between them.

\section{Thermal imaging cameras AGEMA 880 LW and AGEMA 470 Pro SW}

The user's manual of AGEMA $880 \mathrm{LW}$ and AGEMA 470 Pro SW gives simplified formula that describes the atmospheric transmission in infrared, using the LOWTRAN model [2]:

$$
P_{a t m}(d)=\exp \left[-\alpha \cdot\left(\sqrt{d}-\sqrt{d_{c a l}}\right)-\beta \cdot\left(d-d_{c a l}\right)\right]
$$

where: $P_{\text {atm }}$ - atmospheric transmission; $d, \mathrm{~m}$ - camera - object distance; $d_{c a l}, \mathrm{~m}$ - camera-object distance (in calibration process - the value of $1 \mathrm{~m}) ; \alpha, \beta$ coefficients specified for normal conditions: atmospheric temperature $T_{a t m}=15^{\circ} \mathrm{C}$, relative humidity $\omega_{\%}=50 \%$; for short wave bands (SW): $\alpha=0.393$ and $\beta=0.00049$; for long wave bands (LW): $\alpha=0.008$ and $\beta=0$.

The graph of the function (1) for camera LW is presented in fig. 1.

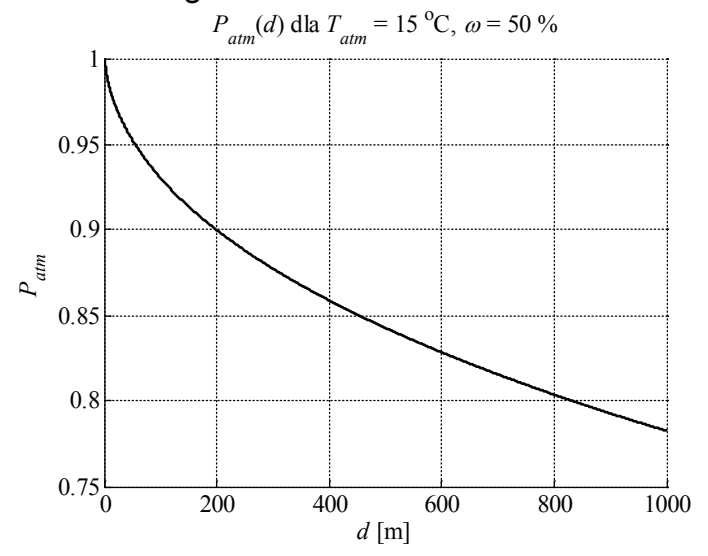

Fig. 1. Characteristics of atmospheric transmission coefficient $P_{a t m}=f(d)$ for AGEMA $880 L W$ as a function of the camera object distanced 
In real conditions it is possible to change only one parameter in formula (1) what causes measurement inaccuracies in calculating coefficient $P_{\text {atm }}$. It directly affects measurement inaccuracies as far as the temperature of the object is concerned which results in inaccuracies of thermovision measurements $[2,3]$.

\section{ThermaCAM PM 595 LW}

In order to estimate the transmission coefficient in ThermaCAM PM 595 LW the FLIR company applied different equations according to formulas (2) and (3). This model is defined for a LW detector with an average wavelength $7.5 \div 13 \mu \mathrm{m}$ [4].

$$
\begin{gathered}
\omega\left(\omega_{\%}, T_{a t m}\right)=\omega_{\%} \cdot \\
\exp \left(h_{1} \cdot T_{a t m}{ }^{3}+h_{2} \cdot T_{a t m}{ }^{2}+h_{3} \cdot T_{a t m}+h_{4}\right) \\
P_{a t m}\left(d, T_{a t m}, \omega\right)=K_{a t m} \cdot \exp \left[-\sqrt{d} \cdot\left(a_{1}+b_{1} \cdot \sqrt{\omega}\right)\right] \\
+\left(1-K_{a t m}\right) \cdot \exp \left[-\sqrt{d} \cdot\left(a_{2}+b_{2} \cdot \sqrt{\omega}\right)\right]
\end{gathered}
$$

$\omega$ - coefficient showing the content of water vapour in the atmosphere; $\omega_{\%}$ - relative humidity; $T_{a t m}{ }^{\circ} \mathrm{C}$ temperature of the atmosphere; $d, \mathrm{~m} ; K_{\mathrm{atm}}=1.9$ scaling factor for the atmosphere damping; $a_{1}, a_{2}, b_{1}, b_{2}$, $h_{1}, h_{2}, h_{3}, h_{4}$ - example values of coefficients, selected experimentally for the temperature range $-40 \div 120^{\circ} \mathrm{C}$ for the thermal imaging camera ThermaCAM PM 595 LW FLIR company, taken from the thermogram file [4]: $a_{1}=0.0066 ; \quad a_{2}=0.0126 ; \quad b_{1}=-0.0023 ; \quad b 2=-0.0067 ;$ $h_{1}=6.8455 \cdot 10^{-7} ; \quad h_{2}=-2.7816 \cdot 10^{-4} ; \quad h_{3}=6.939 \cdot 10^{-2}$; $h_{4}=1.5587$.

In a similar way as for fig. 1, the characteristics were specified using formulas (2), (3) and shown in fig. 2 and 3.

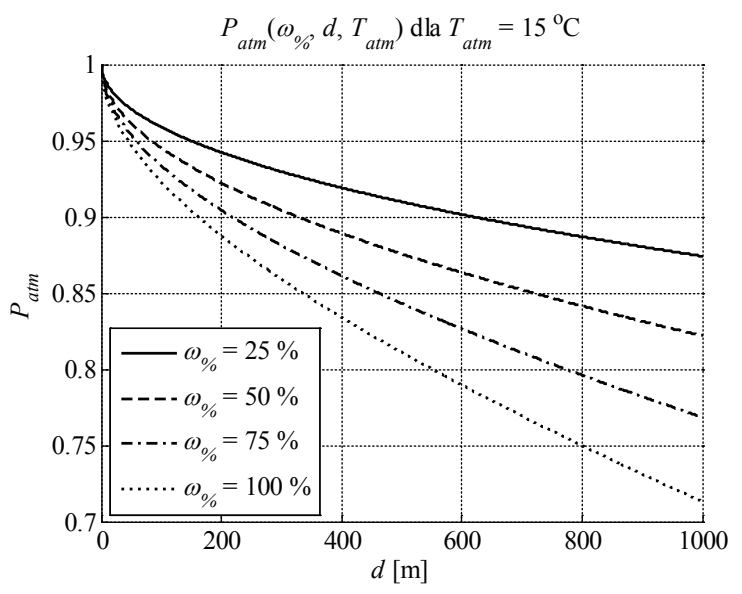

Fig. 2. Characteristics of atmospheric transmission coefficient $P_{a t m}=f(d)$ for long way band LW ThermaCAM PM 595 camera as a function of the relative humidity of the atmosphere $\omega \%$.

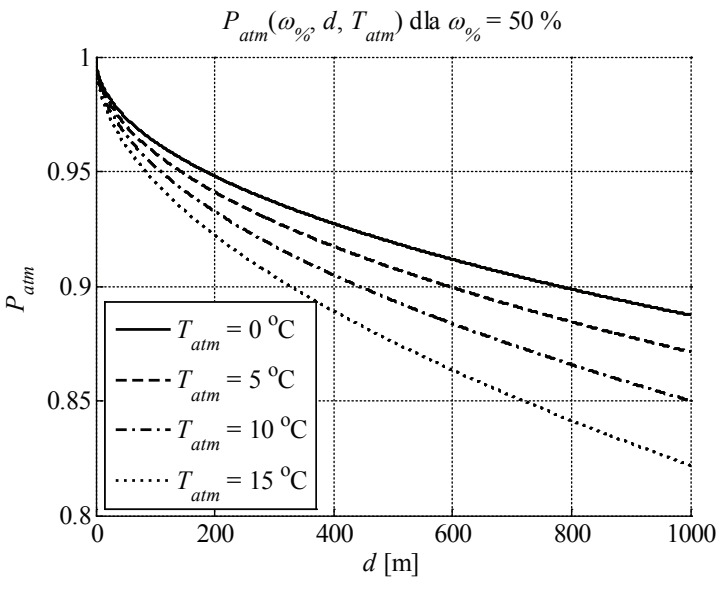

Fig. 3 Characteristics of atmospheric transmission coefficient $P_{a t m}=f(d)$ for long way band LW ThermaCAM PM 595 camera as a function of the temperature of the atmosphere $T_{\text {atm. }}$.

Formula (3) for calculating atmospheric transmission coefficient $P_{\text {atm }}$ includes all parameters affecting thermal measurement, therefore they are universal formulas which can be used in practice by FLIR [4].

\section{Passman - Larmore tables}

Using experimental studies conducted by Passman Larmore [1, 3] the characteristics of the transmission coefficient can be calculated precisely. Gas composition influences the results of measurement carried out using a thermovision camera. In this case, the most important are the absorbance coefficients: vapour absorbance $\left(\mathrm{P}_{\mathrm{H} 2 \mathrm{O}}\right)$ and carbon dioxide absorbance $\left(\mathrm{P}_{\mathrm{CO} 2}\right)$. According to formula (4) we have [1]:

$$
P_{\text {atm }} \cong P_{\mathrm{H}_{2} \mathrm{O}} \cdot P_{\mathrm{CO}_{2}}
$$

The vapour absorbance depends on the number of absorbing molecules, i.e., on the partial pressure of water vapour, and the distance $d$ travelled by radiation in the absorbing medium. It is usually defined as height $h$ of the cylinder with diameter $D$. The volume of cylinder is equal to the volume of liquid obtained by condensation of water vapour contained in the cylinder with diameter $D$ and height $d$, reflecting the atmosphere which is at the distance $d$ where the thermovision camera measurement is taken for $d=1 \mathrm{~km}$. It is also shown in fig. 4. Taking into consideration the formulas described above the following formula can be used (5):

$$
h=\left(4 \cdot V_{\mathrm{H}_{2} \mathrm{O}}\right) /\left(\pi \cdot D^{2}\right)
$$

$V_{H 2 O}$ - volume of liquid obtained by condensation of water vapour in the absorbing medium; $D$ - diameter of the cylinder representing the absorbing medium.

Vapour absorbance $P_{\mathrm{H} 2 \mathrm{O}}$ depends on molecular processes which are responsible for selective absorption spectrum. $P_{H 2 O}$ also depends on temperature and total pressure of the gas mixture which 
regulates the width of absorbing lines as a result of molecular collisions and the Doppler effect.

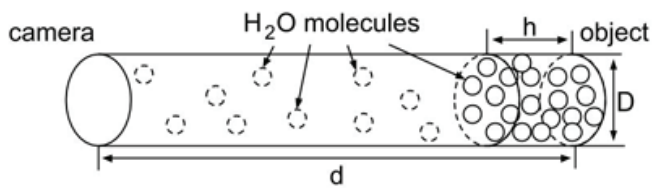

Fig. 4 The figure shows the cylinder with height $h$ and diameter $D$, it includes condensated water vapour and it is in the distance of measurement using thermovision camera [1].

There is a relation between the height of the cylinder with water $h$ - formula (5), temperature $T_{a t m}$ and relative humidity $\omega_{\%}$. An approximation of a graph was obtained using of Newton Method $h(d)$ described in [1] and its dependency on relative humidity and distance was taken into consideration according to formula (6). See the graph in fig. 5 .

$$
\begin{aligned}
& h\left(d, \omega_{\%}\right)=\left(0.00016667 \cdot T_{a t m}{ }^{3}+0.01 \cdot T_{a t m}{ }^{2}+\right. \\
& \left.+0.38333 \cdot T_{a t m}+5\right) \cdot \omega_{\%} \cdot d
\end{aligned}
$$

$h, \mathrm{~mm} / \mathrm{km}$ - height of the cylinder with water; $T_{\text {atm }},{ }^{\circ} \mathrm{C}-$ the ambient temperature; $\omega_{\%}$ - relative humidity; $d, \mathrm{~km}-$ distance.

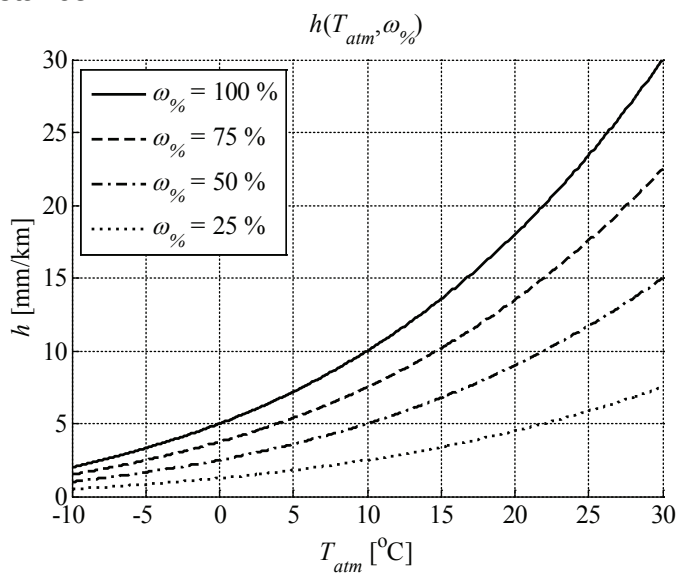

Fig. 5. Characteristics of coefficient $h, \mathrm{~mm} / \mathrm{km}$ for $d=1 \mathrm{~km}$

Taking into account distance $d$, height of the cylinder with water $h$ and wavelength $\lambda$, the appropriate values of $P_{\mathrm{H} 2 \mathrm{O}}$ and $P_{\mathrm{CO} 2}$ can be determined using the Passman - Larmore tables. When these values are placed into formula (4) it is possible to calculate atmospheric transmission coefficient $P_{\text {atm }}$. A part of Passman - Larmore table is shown in table $1[1,3]$. The following example shows how to calculate the atmospheric transmission coefficient.

\section{Example}

Calculate the atmospheric transmission coefficient for: $\lambda$ $=13 \mu \mathrm{m}, d=500 \mathrm{~m}, \omega_{\%}=50 \%,(\omega=0.5)$ and $T_{\text {atm }}=20$ ${ }^{\circ} \mathrm{C}$.
Using formula (6) the result is: $h(d, \omega)=$ $\left(0.00016667 \cdot 20^{3}+0.01 \cdot 20^{2}+0.38333 \cdot 20+5\right) \cdot 0.5 \cdot 0.5=$ $4.5 \approx 5$. When the values are placed in table 1 and 2 , the results are: 0.921 and 0.977 . They are placed in formula (4) and in this way the value of atmospheric transmission coefficient is calculated and equals approximately 0.900 .

In order to adapt the results to models described for AGEMA and ThermaCAM average characteristics for wavelength $\lambda=7.5 \div 13 \mu \mathrm{m}$ are shown in fig. 6 .

Tab. 1. A part of Passman - Larmore table for vapour absorbance $P_{\mathrm{H} 2 \mathrm{O}}[1,3]$

\begin{tabular}{cccccccc}
\hline & \multicolumn{7}{c}{$\mathrm{P}_{\mathrm{H} 2 \mathrm{O}}$} \\
\cline { 2 - 8 }$\lambda, \mu \mathrm{m}, \mathrm{mm} / \mathrm{km}$ \\
\cline { 2 - 8 } & 0.2 & 0.5 & 1 & 2 & 5 & 10 & 20 \\
\hline 7.5 & 0.947 & 0.874 & 0.762 & 0.582 & 0.258 & 0.066 & 0 \\
8.0 & 0.990 & 0.975 & 0.951 & 0.904 & 0.777 & 0.603 & 0.365 \\
9.0 & 0.997 & 0.992 & 0.984 & 0.968 & 0.921 & 0.848 & 0.719 \\
10.0 & 0.998 & 0.994 & 0.988 & 0.975 & 0.940 & 0.883 & 0.780 \\
11.0 & 0.998 & 0.994 & 0.988 & 0.975 & 0.940 & 0.883 & 0.779 \\
12.0 & 0.997 & 0.993 & 0.987 & 0.974 & 0.937 & 0.878 & 0.770 \\
13.0 & 0.997 & 0.992 & 0.984 & 0.967 & 0.921 & 0.846 & 0.718 \\
\hline
\end{tabular}

Tab. 2. A part of Passman - Larmore table for carbon dioxide absorbance $P_{\mathrm{CO} 2}[1,3]$

\begin{tabular}{cccccccc}
\hline & \multicolumn{7}{c}{$\mathrm{P}_{\mathrm{CO} 2}$} \\
\cline { 2 - 8 }$\lambda, \mu \mathrm{m}$ & 0.2 & 0.5 & 1 & 2 & 5 & 10 & 20 \\
\cline { 2 - 8 } & 1.000 & 1.000 & 1.000 & 1.000 & 1.000 & 1.000 & 1.000 \\
\hline 7.5 & 1.000 & 1.000 & 1.000 & 1.000 & 1.000 & 1.000 & 1.000 \\
8.0 & 1.000 & 1.000 & 1.000 & 1.000 & 1.000 & 1.000 & 1.000 \\
9.0 & 1.000 & 1.000 & 0.999 & 0.997 & 0.994 & 0.989 & 0.978 \\
10.0 & 1.000 & 0.999 & 0.999 & 0.997 & 0.993 & 0.986 & 0.973 \\
11.0 & 1.000 & 1.000 & 0.999 & 0.999 & 0.997 & 0.993 & 0.986 \\
12.0 & 0.991 & 0.977 & 0.955 & 0.912 & 0.794 & 0.630 & 0.397 \\
13.0 & 0.970
\end{tabular}

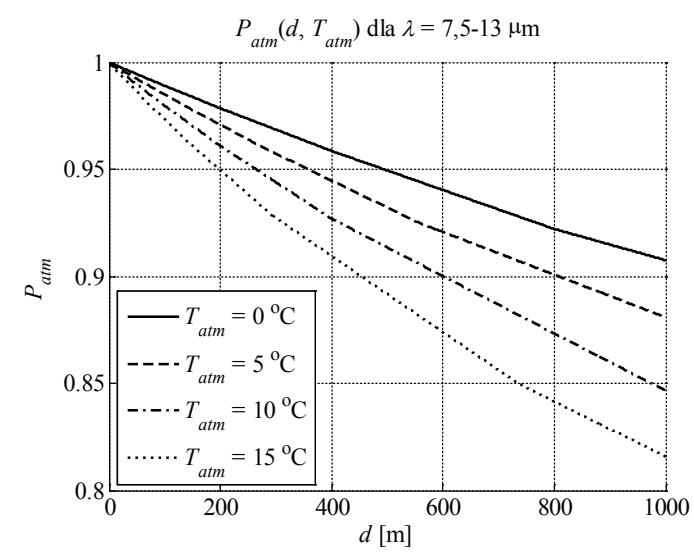

Fig. 6. Characteristics of atmospheric transmission coefficient $P_{a t m}=f(d)$ calculated using Passman - Larmore tables.

The tables were obtained on the basis of experimental studies and that is why the model seems to give the most accurate value of atmospheric transmission 
coefficient $P_{\text {atm }}$. Calculating atmospheric transmission coefficient by means of residual value of $P_{a t m}$ is timeconsuming.

\section{Approach adopted in this paper [5]}

The paper [5] gives one more atmospheric transmission model in infrared $P_{\text {atm. }}$. First the formula (7) describing saturation vapour pressure was introduced:

$$
\begin{aligned}
& p_{s}\left(T_{a t m}\right)= \\
& = \begin{cases}6.112 \cdot \exp \left(21.874 \frac{T_{a t m}-273.17}{T_{a t m}-7.66}\right) & \text { for } T_{a t m}<273.15 \mathrm{~K}(7) \\
6.112 \cdot \exp \left(17.269 \frac{T_{a t m}-273.17}{T_{a t m}-35.86}\right) & \text { for } T_{a t m} \geq 273.15 \mathrm{~K}\end{cases}
\end{aligned}
$$

$p_{s}, \mathrm{~Pa}$ - saturation vapour pressure; $T_{\text {atm }}, \mathrm{K}$ temperature of the atmosphere.

The value of coefficient $\alpha_{\lambda}$ was calculated. It describes cross the section of vapour molecule which depends on wavelength - formula (8). In the paper [5] formula (8) describes correlation resulting from Beer's law and ideal gas law.

$\alpha_{\lambda}=-\left[\left(k_{B} \cdot T_{a t m}\right) /\left(\omega_{\%} \cdot p_{d} \cdot d\right)\right] \cdot \ln \left(P_{a t m}\right)$

$k_{B}=1.28 \cdot 10^{-23}, \mathrm{~J} / \mathrm{K}-$ Boltzmann constant; $T_{\text {atm }}, \mathrm{K} ; \omega_{\%}-$ relative humidity; $p_{s}, \mathrm{~Pa}$ - saturation vapour pressure; $d, \mathrm{~m} ; P_{\text {atm }}$ - the value of atmospheric transmission coefficient resulting from calibration measurements or literature data (e.g. Passman - Larmore tables, see the example above).

Using formulas (7), (8) and assuming constant wavelength $\lambda$, atmospheric transmission coefficient $P_{\text {atm }}$ [5] can be calculated according to the formula:

$$
P_{\text {atm }}\left(d, T_{\text {atm }}, \omega_{\%}\right)=\exp \left(-\alpha_{\lambda} \cdot d \cdot p_{s} \cdot \omega_{0}\right) /\left(k_{B} \cdot T_{a t m}\right)
$$

Calibration value $P_{a t m}$ is $P_{a t m}=0.8160$. The method of calculating this value - see example. It was calculated using Passman - Larmore tables for $d=1000 \mathrm{~m}$, $\omega_{\%}=50 \%, \quad T_{\text {atm }}=15{ }^{\circ} \mathrm{C}$ and $\lambda=7.5 \div 13 \mu \mathrm{m}$. Fig. 7 presents graphs of the coefficient. Formula (9) gives satisfactory results of $P_{\text {atm }}$, but it is a huge obstacle to set calibration point properly.

\section{Comparison graphs}

In order to compare all models describing atmospheric transmission $P_{a t m}$ in infrared analyzed in this paper, the same parameters were applied. The model for AGEMA 880 LW was defined for constant temperature and relative humidity, i.e. $T_{a t m}=15^{\circ} \mathrm{C}$ and $\omega_{\%}=50 \%$. The first two models were defined for long wave bands, where the average wavelength was used. To adapt the scope to Passman - Larmore model tables 1 and 2 in the range $8 \div 14 \mu \mathrm{m}$ and the average result was used. Model (9) shown in this paper [5] depends on the calibration value. The calibration value calculated in associated paragraph and amounted to 0.8160 was used.

\section{Conclusions}

The atmosphere blocks infrared radiation of the object whose temperature is measured. In the case when there is no properly designed model including the atmospheric transmission coefficient in infrared $P_{a t m}=f(d)$ in microcontroller of a thermal imaging camera, the results given by the camera are inappropriate, too low. It is very important to use a good model of atmospheric transmission coefficient $P_{a t m}=f(d)$. The paper has some limitations. Thermal imaging camera models should take into account additional factors affecting on the measurement, such as for example spectral response. The dependence of the atmosphere transmission for various types of thermal imaging cameras matrix cannot be brought into one universal plot. Bearing in mind described limitations in the paper were made only comparison the plots.

The graph based on Passman - Larmore measurements was introduced as the initial model. All models have similar characteristics - see fig. 8 , which become more different with the distance - see fig. 9 . The model described in the paper [5], can be much more different from the others if the calibration point is not appropriate. Practical and experimental models give similar values of atmospheric transmission coefficient $P_{a t m}$. This is due to the fact that the model of the atmospheric transmission coefficient is chosen properly. As a result similar results can be obtained using simple calculations. In fig. 8 and 9 similar conditions were applied for all the models in typical measurement situations. In different circumstances comparative characteristics can be different. This results from the fact that the model described in the paper [5] can be used in practice only when the calibration point is chosen properly. Fig. 9 shows huge disparities in characteristics of atmospheric transmission coefficient $P_{a t m}=f(d)$. This is particularly noticeable for distance over $1.000 \mathrm{~m}$ (fig. 9). For the distance up to $1.000 \mathrm{~m}$ (fig. 8) all characteristics are similar. All these models are dedicated to a certain type of camera. The Passman Larmore model is the most universal, because it is possible to use tables for different wavelength $\lambda$.

It should be noted that properly calculated atmospheric transmission coefficient in infrared $P_{a t m}=\mathrm{f}(d)$ enables to read the right temperature of the object. $A$ correct value of atmospheric transmission coefficient inserted in microcontroller of the camera is of fundamental importance for accuracy of contact-less measurement of the object temperature [2]. Additional information about the atmospheric transmission coefficient in infrared can be found in items $[6,7,8,9]$. 


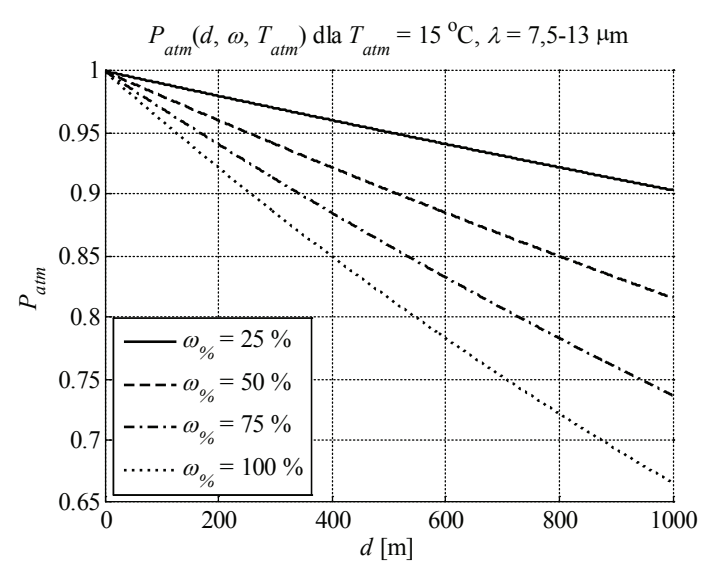

Fig. 7. Characteristics of atmospheric transmission coefficient $P_{\text {atm }}=f(d)$ calculated according to the approach given in [5]

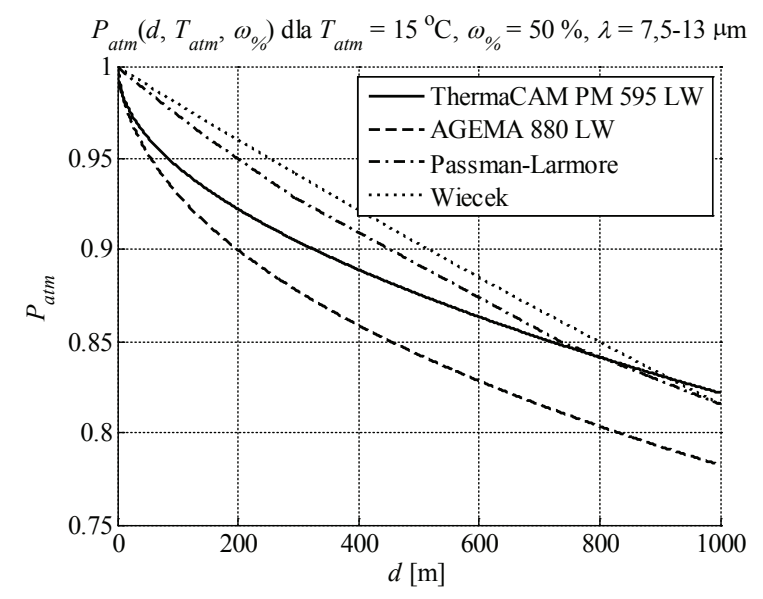

Fig. 8. Characteristics of atmospheric transmission coefficient $P_{a t m}=f(d)$ for a few models described in this paper for the distance up to $1.000 \mathrm{~m}$

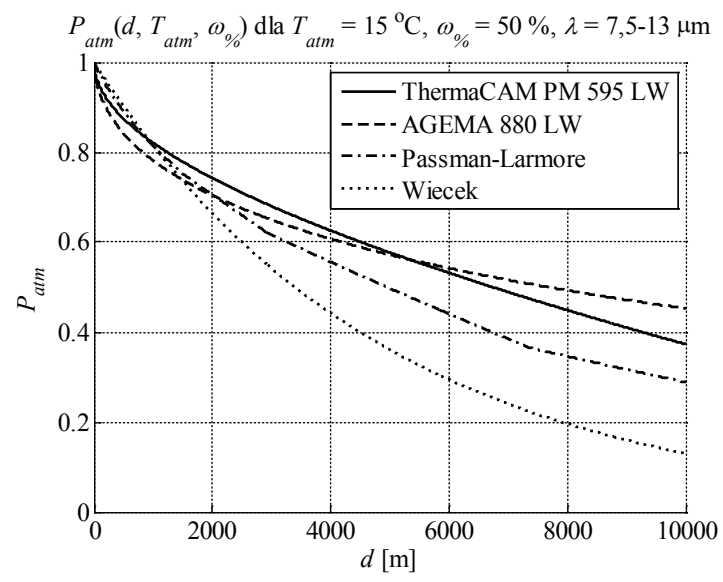

Fig. 9. Characteristics of atmospheric transmission coefficient $P_{a t m}=f(d)$ for a few models described in this paper for the distance up to $10.000 \mathrm{~m}$

\section{References}

[1] Gaussorgues G.: Infrared Thermography. Springer Science+Business Media, B.V., Dordrecht 1994, ISBN 978-94-010-4306-9.

[2] Minkina W., Dudzik S.: „Infrared thermography - errors and uncertainties" John Wiley \& Sons Ltd, Chichester 2009 r., ISBN 978-0-470-74718-6.

[3] Passman S., Larmore L.: Atmospheric Transmission. Rand Paper, Rand Corporation, Santa Monica, 1956, p. 897.

[4] Toolkit IC2 Dig16 Developers Guide 1.01 for Agema 550/570, ThermaCAM PM 5x5 and the ThermoVision Family. FLIR Systems 2001 - in the daytime: 20.02.2014, http://www.workswell.cz/manuals/flir/hardware/Toolkit IC 2 Dig 16/Toolkit IC2 Dig16 Developers Guide $101 \mathrm{~A}$ GEMA 550570 ThermaCAM PM5X5 and the Therm oVisionFamily.pdf.

[5] Więcek B.: The influence of water vapour on infrared atmospheric transmission. Pomiary Automatyka Komputery w Gospodarce i Ochronie Środowiska, 2008 Nr 2, pp. 15-18 (in Polish).

[6] Pręgowski P.: „The analysis of signal and its influence on construction of thermographic devices", Ph. D. Thesis, Institute of Optoelectronics, Military University of Technology, Warszawa, 1985 (in Polish).

[7] Anderson G. P., Kneizys F. X., Chetwynd J.H., Wang J., Hoke M. L. and others: "FASCODE, MODTRAN, LOWTRAN: past, present, future" Proceedings of the $18^{\text {th }}$ Annual Review Conference on Atmospheric Transmission Models, Boston 06-08.06.1995, pp. 101-120, editors: Anderson G. P., Picard R. H., Chetwynd J. H.

[8] Rothman L. S., Jacquemart D., Barbe A., Benner D. Ch. and others: „The HITRAN 2004 molecular spectroscopic database" Journal of Quantitative Spectroscopy and Radiative Transfer, 96 (2005) pp. 139-204.

[9] Narasimhan S. G., Nayar S. K.: „Vision and the atmosphere" International Journal of Computer Vision 48 (3) 2002, pp. 233-254. 